\title{
A Contrast on Conductor Galloping Amplitude Calculated by Three Mathematical Models with Different DOFs
}

\author{
Bin Liu, ${ }^{1}$ KuanJun Zhu, ${ }^{1}$ XiaoQin Sun, ${ }^{2,3}$ Bing Huo, ${ }^{2,3}$ and XiJun Liu ${ }^{2,3}$ \\ ${ }^{1}$ China Electric Power Research Institute, Beijing 100029, China \\ ${ }^{2}$ School of Mechanical Engineering, TianJin 300072, China \\ ${ }^{3}$ TianJin Key Laboratory of Nonlinear Dynamics and Chaos Control, TianJin 300072, China \\ Correspondence should be addressed to Bin Liu; liubinliubin@yeah.net
}

Received 27 February 2014; Revised 5 July 2014; Accepted 6 July 2014; Published 21 July 2014

Academic Editor: Vadim V. Silberschmidt

Copyright (C) 2014 Bin Liu et al. This is an open access article distributed under the Creative Commons Attribution License, which permits unrestricted use, distribution, and reproduction in any medium, provided the original work is properly cited.

It is pivotal to find an effective mathematical model revealing the galloping mechanism. And it is important to compare the difference between the existing mathematical models on the conductor galloping. In this paper, the continuum cable model for transmission lines was proposed using the Hamilton principle. Discrete models of one DOF, two DOFs, and three DOFs were derived from the continuum model by using the Garlekin method. And the three models were compared by analyzing the galloping vertical amplitude and torsional angle with different influence factors. The influence factors include wind velocity, flow density, span length, damping ratio, and initial tension. The three-DOF model is more accurate at calculating the galloping characteristics than the other two models, but the one-DOF and two-DOF models can also present the trend of galloping amplitude change from the point view of qualitative analysis. And the change of the galloping amplitude relative to the main factors was also obtained, which is very essential to the antigalloping design applied in the actual engineering.

\section{Introduction}

Conductor galloping is a typical self-excited aeroelastic vibration phenomenon [1]. This conductor motion is characterized by large amplitude (possibly $>10 \mathrm{~m}$ ) and low frequency (approximately $0.1-3 \mathrm{~Hz}$ ) [2]. Galloping can cause various kinds of structural and electrical damages for overhead transmission lines $[3,4]$. Therefore, it has become a difficult problem for transmission technology and excited more and more attention in the world.

Conductor galloping represents a classical motion instability mechanism in the steady flow over a noncircular cross section caused by ice accretion on the conductor. It is pivotal to find an effective mathematical model to reveal the galloping mechanism. The galloping models have been studied extensively over a half century since den Hartog [5] firstly established the vertical galloping mechanism based on a simple single degree-of-freedom (DOF) spring-oscillator model. And the quasisteady hypothesis (QSH) was applied to describe the nonlinear aerodynamic forces, whereafter
Nigol and Buchan $[6,7]$ proposed a galloping torsional mechanism based on a two-DOF torsion-oscillator model coupled by vertical and torsional oscillations. They proved that torsional oscillation sometimes plays a crucial role to excite the galloping phenomenon, especially for the bundled conductors. Then, Yu et al. $[8,9]$ obtained a three-DOFs oscillator model coupled by vertical, horizontal, and torsional oscillations. They found that horizontal oscillation has an important influence on the galloping critical conditions and galloping characteristics. But these models are all based on the simple spring-oscillation simulation for iced conductor cross section in two-dimensional space. Some important factors, such as span length, initial tension, and sag, cannot be considered in these models.

In order to remedy the above disadvantages of the simple oscillator models, the cable modeling method was proposed in recent years. And the models for transmission lines have been studied in many works, which can also be classified as a continuum mathematical model. Luongo et al. $[10,11]$ and Lee and Perkins [12] established a flexible cable model which 


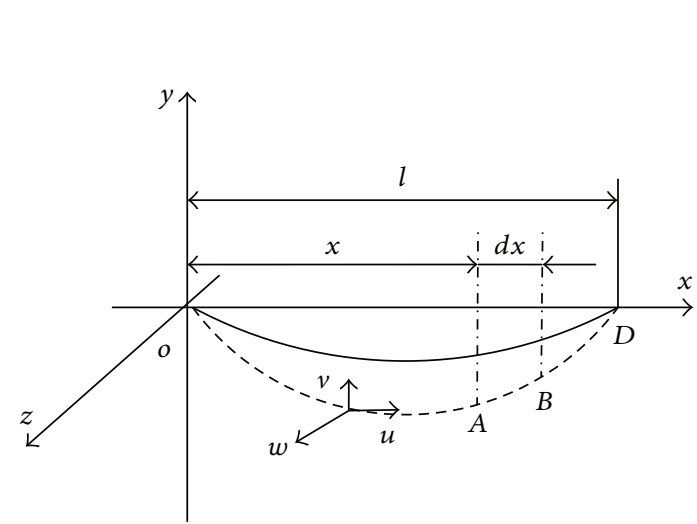

(a)

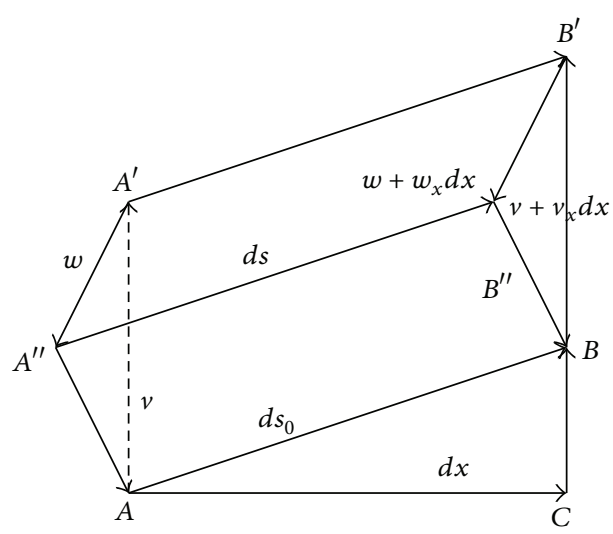

(b)

FIGURE 1: Model of the transmission line: (a) cable model and (b) infinitesimal element.

only accounts for the static rotation. Yu et al. [9], McConnell and Chang [13], and White et al. [14] employed a cablebeam model, accounting for twisting but not for bending and neglecting the cable initial curvature in defining the torsion strain. Because the bending moment is not taken into account in these models, the moment equilibrium around the tangent to the cable is violated [15]. So a consistent cablebeam model has become a matter of great interest, which can be able to take into account all the factors $[16,17]$. Recently, Luongo et al. [18-20] have formulated consistent linear and nonlinear models of cable-beam accounting for both torsion and bending. In order to apply in the actual engineering easily, Qin et al. [21] constructed one-DOF, twoDOF, and three-DOF models from the continuum models of transmission lines by using the Hamilton principle. But the comparison of the three models is not studied, which is very important in the application of these models.

In this paper, the continuum cable model for transmission lines was proposed using the Hamilton principle, where the initial displacement, the geometric nonlinearity caused by the large deformation, and the aerodynamic nonlinearity caused by flow were considered. And discrete models of one DOF with vertical motion, two DOFs with vertical-torsional coupling motion, and three DOFs with horizontal-verticaltorsional coupling motion were derived from the continuum model by using the Galerkin method. In order to compare the three models, the galloping amplitude was analyzed by the different factors including wind velocity, flow density, span length, damping ratio, and initial tension. The difference in the three models was proposed by comparing the vertical amplitude and the torsional angle caused by the conductor galloping. And the change of the galloping amplitude relative to the influence factors was also presented, which is much important to the antigalloping design applied in the actual engineering.

\section{Construction of the Models}

2.1. Cable Model. Figure 1(a) shows the schematic diagram of the transmission line under initial tension $T_{0} . l$ is the span length. $u, v$, and $w$ are the longitudinal, vertical, and horizontal displacements of an arbitrary point $A$ in the line, respectively. The static balance state of the transmission line is selected as the initial position. The displacement of an arbitrary infinitesimal element $d x$ in the line is described schematically in Figure 1(b). $A B$ is the initial position with the static arc length $d s_{0}$ and $A^{\prime \prime} B^{\prime \prime}$ is the movement position with the changed arc length $d s$ taken from the line at time $\Delta t$. Suppose that the displacements of the start and the end in longitudinal $(x)$, horizontal $(z)$, and vertical $(y)$ directions are $u(x, t), w(x, t)$, and $v(x, t)$ and $u+u_{x} d x, w+w_{x} d x$, and $v+v_{x} d x$, respectively, where the right subscript " $x$ " indicates differentiation with respect to coordinate $x$.

The initial arc length vector of the line infinitesimal element can be described as

$$
d \overrightarrow{s_{0}}=d x \vec{i}+d y \vec{j}+d z \vec{k},
$$

where $\vec{i}, \vec{j}$, and $\vec{k}$ are the unit vectors of $x, y$, and $z$ directions.

Then, arc length vector after movement can be derived as

$$
\begin{aligned}
d \vec{s}= & d \overrightarrow{s_{0}}+\overrightarrow{B B^{\prime}}-\overrightarrow{A A^{\prime}}+\overrightarrow{B^{\prime} B^{\prime \prime}}-\overrightarrow{A^{\prime} A^{\prime \prime}}=\left(d x+u_{x} d x\right) \vec{i} \\
& +\left(d y+v_{x} d x\right) \vec{j}+w_{x} d x \vec{k},
\end{aligned}
$$

where $\vec{k}$ is the unit vector of $z$ direction.

Using arc length formula, the arc length expressions of the start and end in the movement can be described as

$$
\begin{gathered}
d s_{0}=\sqrt{1+y_{0 x}^{2}} d x, \\
d s=\sqrt{\left(1+u_{x}\right)^{2}+\left(y_{0 x}+v_{x}\right)^{2}+w_{x}^{2}} d x,
\end{gathered}
$$

where $y_{0 x}$ is the initial configuration of the line under the initial tension. And $y_{0 x}$ can be expressed simply using catenary shape function as [22]

$$
y_{0}=-\frac{2 \sigma_{0}}{\gamma} \operatorname{sh} \frac{\gamma x}{2 \sigma_{0}} \operatorname{sh} \frac{\gamma(l-x)}{2 \sigma_{0}},
$$




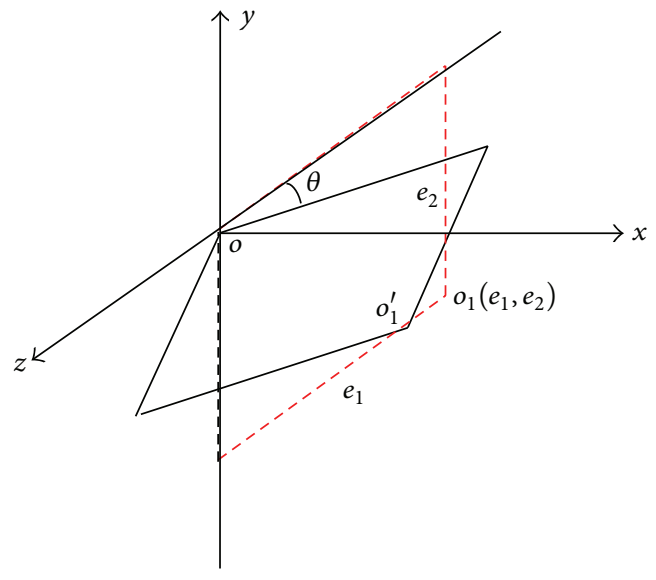

(a)

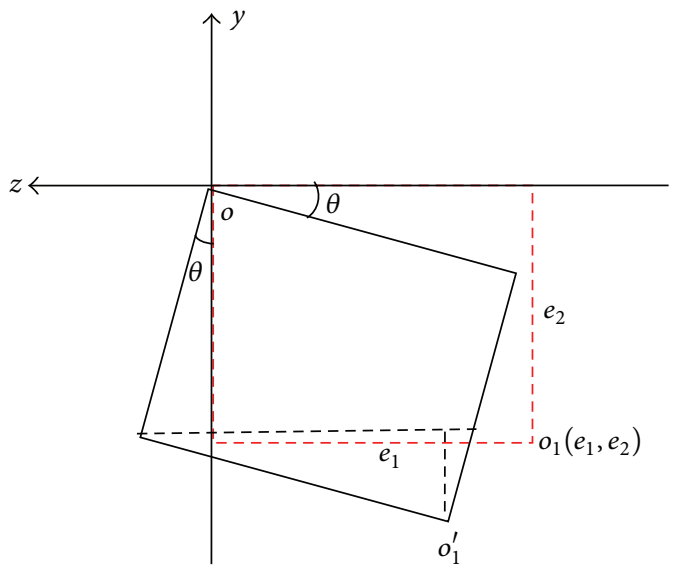

(b)

FIGURE 2: Coordinate system: (- -) original location, (-) location after torsion.

where $\sigma_{0}$ is the axial stress at the lowest position of the line and $\gamma$ is combined load in the line.

Suppose that $d y_{0 x}^{2}$ can be omitted compared to 1 and $u_{x}$ can be omitted compared to $v_{x}$ and $w_{x}$. Using power series expansion and omitting the terms higher than fourth order, the line element strain can be derived from the expressions (1)-(4):

$$
\begin{aligned}
\varepsilon= & \frac{d s-d s_{0}}{d s_{0}}=y_{0 x} v_{x}-y_{0 x}^{3} v_{x}+\frac{1}{4} y_{0 x}^{5} v_{x} \\
& -y_{0 x}^{2} v_{x}^{2}+\frac{1}{2} w_{x}^{2}-\frac{1}{2} y_{0 x}^{2} w_{x}^{2}+\frac{3}{8} y_{0 x}^{4} v_{x}^{2}-\frac{1}{8} v_{x}^{4} \\
& +\frac{1}{16} y_{0 x}^{2} v_{x}^{4}-\frac{1}{2} y_{0 x} v_{x}^{3}+\frac{1}{4} y_{0 x}^{3} v_{x}^{3}+\frac{1}{16} y_{0 x}^{2} w_{x}^{4} \\
& -\frac{1}{2} y_{0 x} v_{x} w_{x}^{2}+\frac{1}{4} y_{0 x}^{3} v_{x} w_{x}^{2}+\frac{1}{8} y_{0 x}^{2} v_{x}^{2} w_{x}^{2} \\
& -\frac{1}{8} w_{x}^{4}+\frac{1}{2} v_{x}^{2}+\frac{1}{8} y_{0 x}^{4} w_{x}^{2}-\frac{1}{4} v_{x}^{2} w_{x}^{2} .
\end{aligned}
$$

2.2. Torsional Motion. It is well known that the torsional displacements have a considerable effect on the instability of the conductor galloping. Especially for bundled conductors, many observations and studies verified that the torsional motion is an integral part of the galloping phenomenon and the initiation of this vibration is exactly based on the coupling between the translational motion and the torsional motion. So the torsional motion is also an essential factor to analyze the conductor galloping. Then, the torsional motion is considered as in Figure 2 [21].

Assuming that $e_{1}$ is the eccentricity in $z$ direction and $e_{2}$ is the eccentricity in $y$ direction, then the displacements of the center of mass are

$$
\begin{gathered}
v=\bar{v}+e_{1} \sin \theta+e_{2} \cos \theta, \\
w=\bar{w}+e_{1} \cos \theta-e_{2} \sin \theta,
\end{gathered}
$$

where $\bar{v}$ and $\bar{w}$ are the displacements of $y$ direction and $z$ direction, respectively.

For $\theta$ is small, (6) can be simplified as

$$
\begin{aligned}
v & =\bar{v}+e_{1} \theta+e_{2}, \\
w & =\bar{w}+e_{1}-e_{2} \theta .
\end{aligned}
$$

And the torsional strain can be defined as

$$
\varepsilon_{\theta}=\frac{\partial \theta}{\partial x}
$$

2.3. Aerodynamic Force Model. Figure 3 shows a typical shape of a crescent-shaped ice accretion on the conductor surface, which is observed frequently in the case of freezing rain. In this figure, $U$ is the absolute wind velocity perpendicular to the conductor axis in the horizontal direction. $F_{D}, F_{L}$, and $M$ are the aerodynamic lift, drag, and moment due to wind action, respectively. $\theta$ is the torsion angle due to the moment on the conductor. $\theta_{0}$ is the initial static off-set angle, namely, the initial ice accretion position on the conductor. $\alpha_{1}$ is the instantaneous angle of attack with respect to the wind, which has the expression

$$
\alpha_{1}=\tan \frac{\dot{v}+R \dot{\theta} \cos \theta}{U-\dot{\omega}+R \dot{\theta} \sin \theta} \approx \frac{\dot{v}+R \dot{\theta}}{U},
$$

where a dot superscript indicates differentiation with respect to time $t, \dot{v}$ is the conductor velocity in the vertical direction, and $\dot{\theta}$ is the angular velocity of the conductor.

Then the dynamic angle of attack $\alpha$ can be expressed based on the quasisteady theory [23]:

$$
\alpha=\theta_{0}+\theta-\frac{\dot{v}+R \dot{\theta}}{U} .
$$

This quasisteady theory assumes that the aerodynamic forces acting on the iced conductor at any instant in motion are identical to that on the stationary conductor under the 


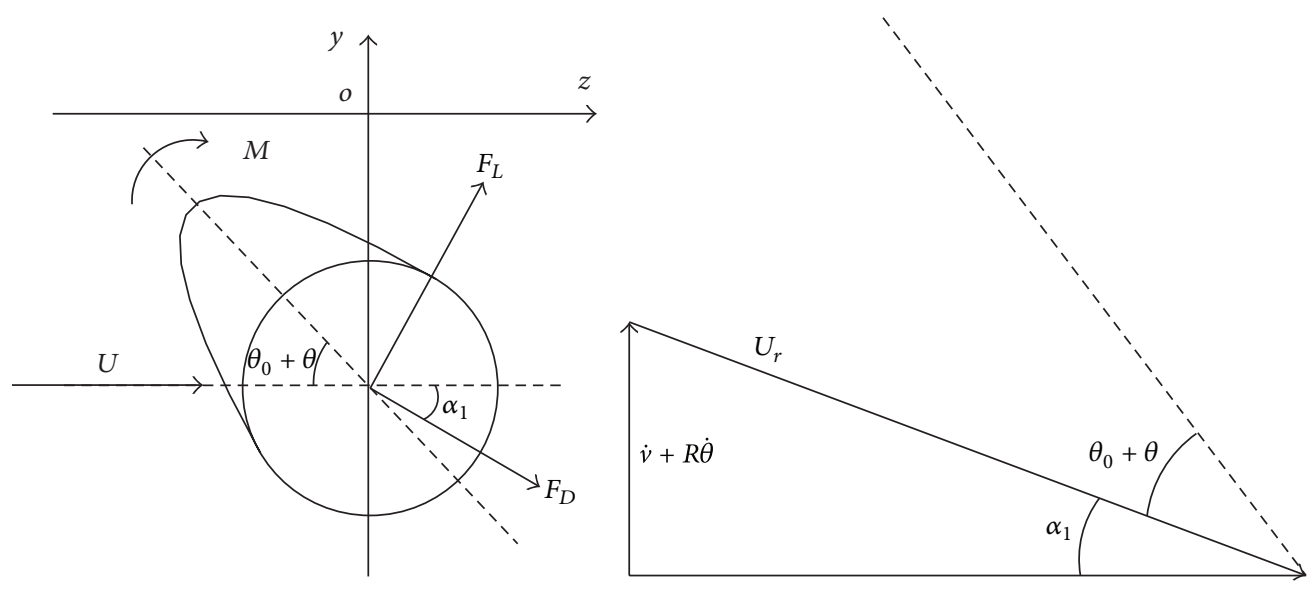

FIgURE 3: The aerodynamic model of iced conductor.

same flow condition. And the use of the quasisteady theory is justified because the frequencies of conductor galloping are much lower than the vortex shedding frequencies. So the curves of the aerodynamic coefficients are obtained by the wind tunnel tests based on the stationary iced conductors at different angles of incidences. In order to establish the aerodynamic model conveniently, the polynomial curve is applied to fit onto the force coefficients versus the dynamic angle of attack by using the experimental data. Then drag coefficient $C_{D}$, lift coefficient $C_{L}$, and moment coefficient $C_{M}$ can be expressed as

$$
C_{L}=\sum_{i=1}^{k} c_{1 i} \alpha^{i}, \quad C_{D}=\sum_{i=1}^{k} c_{2 i} \alpha^{i}, \quad C_{M}=\sum_{i=1}^{k} c_{3 i} \alpha^{i}
$$

where $c_{1 i}, c_{2 i}$, and $c_{3 i}$ are the fitting coefficients of the lift, drag, and moment coefficient curves, respectively, and $k$ is the order of the polynomial. In this study, without loss of generality, $k=3$ is selected for the subsequent analysis. Actually, in the major existing literatures, the third-order polynomial fitting formula is most widely used.

Then the aerodynamic forces have the following expressions:

$$
\begin{gathered}
F_{D}=\frac{1}{2} \rho U^{2} D C_{D}, \quad F_{L}=\frac{1}{2} \rho U^{2} D C_{L}, \\
M=\frac{1}{2} \rho U^{2} D^{2} C_{M},
\end{gathered}
$$

where $\rho$ is the density of the flow and $D$ is the reference length of the iced cross section that is usually substituted by the conductor diameter. And the aerodynamic forces in $y$ and $z$ directions are

$$
F_{y}=F_{L} \cos \alpha_{1}-F_{D} \sin \alpha_{1}, \quad F_{z}=F_{L} \sin \alpha_{1}+F_{D} \cos \alpha_{1} .
$$

2.4. Model with Three DOFs. Based on Sections 2.1, 2.2, and 2.3, the model of conductor galloping with three DOFs (out of plan) can be constructed by using the Hamilton principle [24], which is

$$
\int_{t_{1}}^{t_{2}}(\delta T-\delta V+\delta W) d t=0
$$

where $\delta$ is the first order variational operator, $T$ and $V$ are the total kinetic and strain energies, respectively, and $W$ is the work done by the nonconservative forces. And, based on the description in Sections 2.1 and 2.2, the T, $V$, and $W$ are given by

$$
\begin{gathered}
V=\int_{0}^{l}\left(T_{0}+\frac{1}{2} E A \varepsilon\right)\left(d s-d s_{0}\right)+\int_{0}^{l} \frac{1}{2} G J \varepsilon_{\theta}^{2} d s, \\
T=\int_{0}^{l} \frac{1}{2} m \dot{v}^{2} d s_{0}+\int_{0}^{l} \frac{1}{2} m \dot{w}^{2} d s_{0}+\int_{0}^{l} \frac{1}{2} I \dot{\theta}^{2} d s_{0}, \\
W=\int_{0}^{l}\left(F_{y}-c_{y} \dot{v}\right) v d s_{0}+\int_{0}^{l}\left(F_{z}-c_{z} \dot{w}\right) w d s_{0} \\
+\int_{0}^{l}\left(M-c_{\theta} \dot{\theta}\right) \theta d s_{0},
\end{gathered}
$$

where $m$ is the mass per unit length, $E$ is the elastic modulus, $A$ is the cross section area, $G$ is the shear modulus, $J$ is the moment of inertia, $I$ is polar moment of inertia, and $c_{y}, c_{z}$, and $c_{\theta}$ are the damping coefficients.

Substituting Equations (1)-(13) and (15) into (14) and omitting axial inertial force, the line motion equations can be obtained. Then, the continuum motion equations can be approximated by the Galerkin procedure. In the displacement expansion, the use of the eigenfunctions is often the best choice in the aspects of convergence and accuracy $[11,19,25]$. But in order to concentrate on the study of the comparison of the different models, a simplified Galerkin procedure is presented by using a sine series of the symmetric in-plane 
modes as assumed shape functions [26]. So the displacements $v, w$, and $\theta$ can be expressed as

$$
\begin{gathered}
v(x, t)=q_{v}(t) \sin \frac{\pi}{l} x, \quad w(x, t)=q_{w}(t) \sin \frac{\pi}{l} x, \\
\theta(x, t)=q_{\theta}(t) \sin \frac{\pi}{l} x .
\end{gathered}
$$

Then, employing the Galerkin procedure, the motion equations of conductor galloping can be discretized to a three-DOF model. And neglecting the terms higher than third order, the expression is derived as

$$
\begin{aligned}
& b_{101} \ddot{q}_{v}+\left(b_{102}+c_{y}\right) \dot{q}_{v}+b_{103} q_{v}=F_{1}\left(q_{v}, \dot{q}_{v}, q_{w}, \dot{q}_{w}, q_{\theta}, \dot{q}_{\theta}\right), \\
& b_{201} \ddot{q}_{w}+\left(b_{202}+c_{z}\right) \dot{q}_{w}+b_{203} q_{w}=F_{2}\left(q_{v}, \dot{q}_{v}, q_{w}, \dot{q}_{w}, q_{\theta}, \dot{q}_{\theta}\right), \\
& b_{301} \ddot{q}_{\theta}+\left(b_{302}+c_{\theta}\right) \dot{q}_{\theta}+b_{303} q_{\theta}=F_{3}\left(q_{v}, \dot{q}_{v}, q_{w}, \dot{q}_{w}, q_{\theta}, \dot{q}_{\theta}\right),
\end{aligned}
$$

where $F_{1}-F_{3}$ are the functions relative to $q_{v}, \dot{q}_{v}, q_{w}, \dot{q}_{w}, q_{\theta}$, and $\dot{q}_{\theta}$ in which the maximum order is not greater than three and $b_{101}-b_{103}, b_{201}-b_{203}$, and $b_{301}-b_{303}$ are the integration constants.

2.5. Model with Two DOFs ( $y-\theta$ Direction). The motion in the horizontal direction is much smaller than in the vertical direction for the conductor galloping movement trajectory from the field observations in transmission lines. So, in some studies, the horizontal motion is neglected in the galloping model and only the two DOFs of vertical and torsion are considered.

As a result, when the horizontal DOF is ignored, the expression (5) is changed into

$$
\begin{aligned}
\varepsilon= & \frac{d s-d s_{0}}{d s_{0}}=y_{0 x} v_{x}-y_{0 x}^{3} v_{x}+\frac{1}{4} y_{0 x}^{5} v_{x}+\frac{1}{2} v_{x}^{2}-\frac{1}{8} v_{x}^{4} \\
& -y_{0 x}^{2} v_{x}^{2}+\frac{3}{8} y_{0 x}^{4} v_{x}^{2}-\frac{1}{2} y_{0 x} v_{x}^{3}+\frac{1}{4} y_{0 x}^{3} v_{x}^{3}+\frac{1}{16} y_{0 x}^{2} v_{x}^{4} .
\end{aligned}
$$

And (17) is changed into

$$
\begin{aligned}
& b_{1} \ddot{q}_{v}+\left(b_{2}+c_{y}\right) \dot{q}_{v}+b_{3} q_{v}=F_{4}\left(q_{v}, \dot{q}_{v}, q_{\theta}, \dot{q}_{\theta}\right), \\
& c_{1} \ddot{q}_{\theta}+\left(c_{2}+c_{\theta}\right) \dot{q}_{\theta}+c_{3} q_{\theta}=F_{5}\left(q_{v}, \dot{q}_{v}, q_{\theta}, \dot{q}_{\theta}\right),
\end{aligned}
$$

where $F_{4}$ and $F_{5}$ are the functions relative to $q_{v}, \dot{q}_{v}, q_{\theta}$, and $\dot{q}_{\theta}$ in which the maximum order is not greater than three and $b_{1}-b_{3}$ and $c_{1}-c_{3}$ are the integration constants.

2.6. Model with One DOF ( $y$ Direction). In some studies, only the vertical vibration is considered in order to discuss the galloping characteristics more conveniently for the more complex nonlinear dynamic problem after the conductor galloping has appeared. Actually, the main damages caused by the conductor galloping are attributed to the large amplitude vibration in the vertical direction. So when only the vertical DOF is considered, the expression (10) of the dynamic angle of attack is changed into

$$
\alpha=\theta_{0}-\frac{\dot{v}}{U_{z}} .
$$

TABLE 1: Parameter employed in this analysis.

\begin{tabular}{lccc}
\hline Parameters & Notation & Units & Data \\
\hline Wind velocity & $U$ & $\mathrm{~m} / \mathrm{s}$ & 9 \\
Elastic modulus & $E$ & $\mathrm{GPa}$ & 47.8 \\
Polar moment of inertia & $I$ & $\mathrm{Kg} \cdot \mathrm{m}\left(10^{-3}\right)$ & 0.3334 \\
Diameter of conductor & $D$ & $\mathrm{~mm}$ & 28.6 \\
Horizontal initial tension & $T_{0}$ & $\mathrm{kN}$ & 30 \\
Span length & $l$ & $\mathrm{~m}$ & 125.88 \\
Cross-sectional area & $A$ & $\mathrm{~mm}{ }^{2}$ & 423.24 \\
Mass of unit length & $m$ & $\mathrm{~kg} / \mathrm{m}$ & 2.3789 \\
Torsional stiffness & $G J$ & $\mathrm{~N} \cdot \mathrm{m}^{2} / \mathrm{rad}$ & 101 \\
Initial ice angle & $\theta$ & $\circ$ & 40 \\
& $\xi_{y}$ & & 0.04 \\
Damping ratio & $\xi_{z}$ & & 0.02 \\
& $\xi_{\theta}$ & & 0.06 \\
\hline
\end{tabular}

And (17) is changed into

$$
\begin{aligned}
b_{01} \ddot{q}_{v}+\left(b_{02}+c_{y}\right) \dot{q}_{v}+b_{03} q_{v}= & b_{04} q_{v}^{2}+b_{05} q_{v}^{3} \\
& +b_{06} \dot{q}_{v}^{2}+b_{07} \dot{q}_{v}^{3},
\end{aligned}
$$

where $b_{01}-b_{07}$ are the integration constants.

\section{Contrast Analysis of Different Models on Galloping Amplitude}

In this section, without loss of generality, a typical conductor is selected to analyze the galloping characteristics by using the different models under the different factors. And parameters of the line are tabulated in Table 1. The fitting lift coefficients are $c_{11}=-0.1667, c_{12}=-4.0547$, and $c_{13}=8.3581$. The drag coefficients are $c_{21}=-0.8605, c_{22}=0.8325$, and $c_{23}=1.7815$. And the moment coefficients are $c_{31}=-0.7272, c_{32}=0.2935$, and $c_{33}=5.9704$.

There are numerous methods to analyze the nonlinear dynamic equations, which can be divided into two classes, qualitative methods and quantitative methods. Qualitative methods include Multiple Scales Perturbation Method, Harmonic Balance Method, and Cell Mapping. Numerical integration method which is used to solve the galloping equations can be considered as a quantitative method, which may obtain more accurate results [27].

The focus of this study is on the comparison of the difference of three models. So only a direct numerical integration method used to obtain the galloping amplitude is selected in this study. And the numerical integration is based on the fourth-order Runge-Kutta method. After specifying initial conditions, the integration is carried out until the system reaches a steady state, determined by checking the constancy of the peak-to-peak amplitudes over several consecutive periods.

3.1. Influence of the Wind Velocity on the Galloping Amplitude. Figure 4 shows the galloping amplitudes $q_{v}$ and $q_{\theta}$ versus the horizontal wind velocity $U$ based on three different 


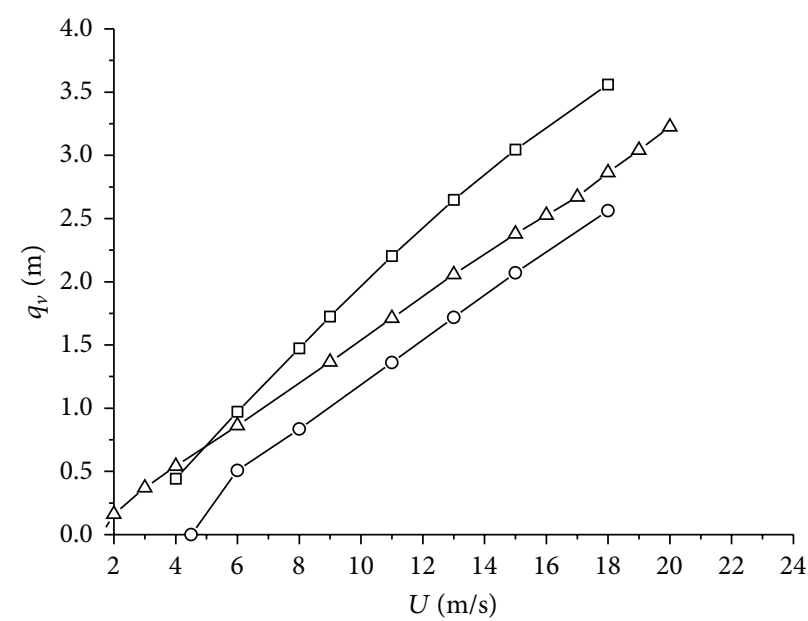

$-\square-$ 1D model
$-\circ-2 \mathrm{D}$ model
$-\Delta-3 \mathrm{D}$ model

(a)

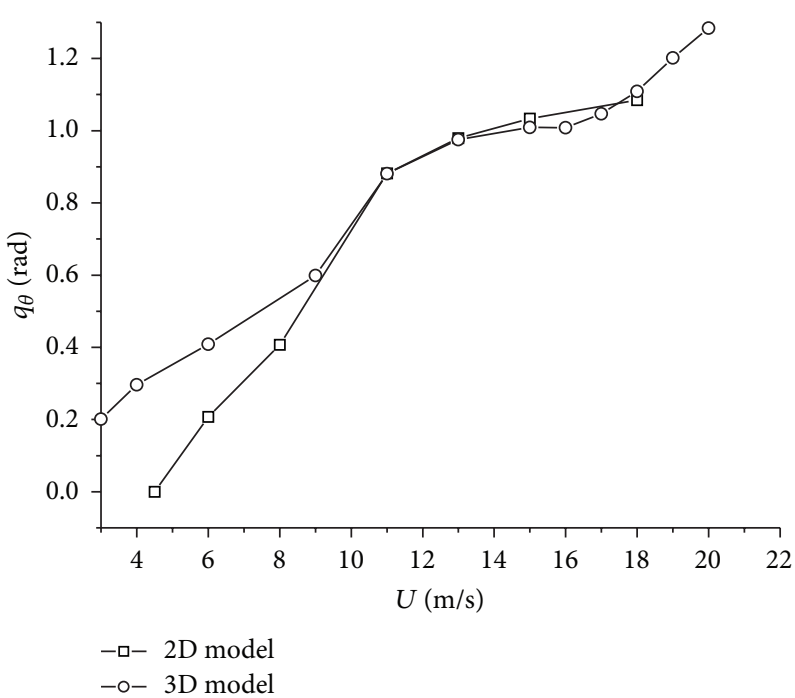

(b)

FIGURE 4: The influence of the velocity on the galloping amplitude: (a) vertical amplitude and (b) torsional amplitude.

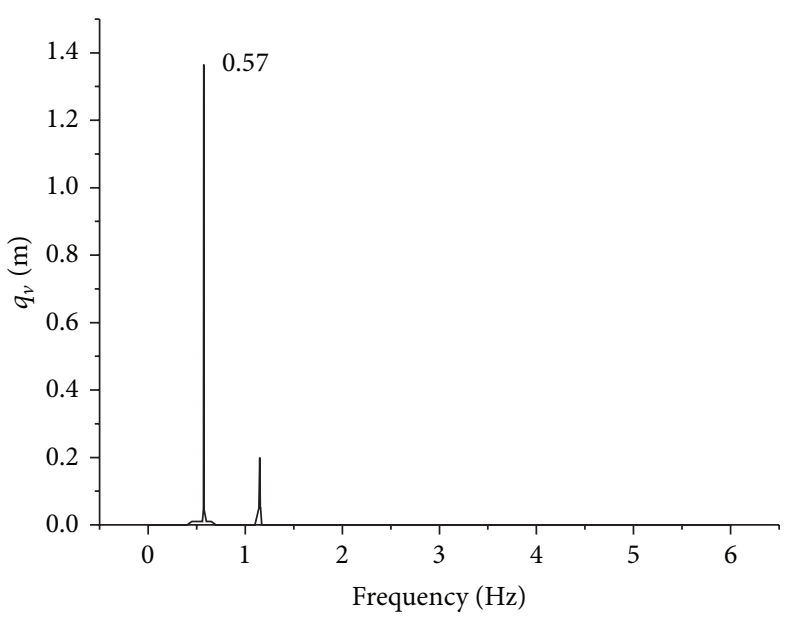

(a)

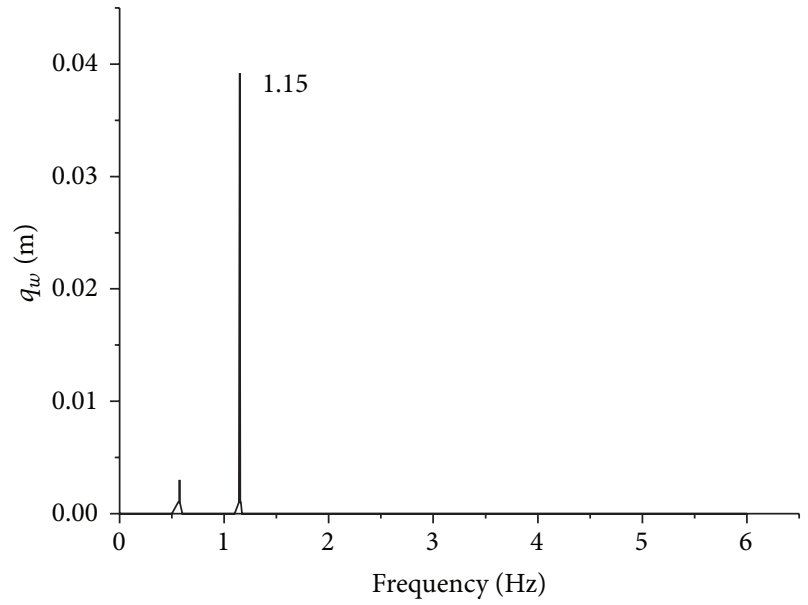

(b)

FIGURE 5: The amplitude-frequency spectrum of numerical solutions $(U=9 \mathrm{~m} / \mathrm{s})$.

models. From Figure 4(a), it is obvious that there are the same trends of the galloping amplitude relative to the wind velocity variation based on the three models. After the galloping phenomenon starts, the galloping amplitude increases as the wind velocity increases. At the same wind velocity, the galloping amplitude calculated by the one-DOF model is much larger than the other models, and the amplitude calculated by the two-DOF model is the smallest. From the results, we can see that the coupling of the horizontal and torsional motions with the vertical motion will reduce the galloping amplitude under the condition of the same wind velocity. And the amplitude calculated by the three-DOF model is larger than the two-DOF model because of the effect of the horizontal motion.

From Figure 4(b), torsional angles obtained by two-DOF and three-DOF models will all increase as the wind velocity increases. And the torsional angles will tend to be equal to the much higher wind velocity. So it has an obvious influence of the horizontal motion to the torsional angle in the lower wind velocity.

From the amplitude responses obtained by the purely numerical procedure, it can be approximately identified as the amplitude corresponding to the leading frequencies in $1: 2$ ratio under the given wind velocity, which is consistent with some other literatures [11]. In addition, it is not difficult to obtain that the squared ratio between the transversal frequency and the torsional frequency is small, which is in accord with the torsional theory for the conductor galloping phenomenon. Figure 5 presents the amplitudefrequency spectrum of numerical solutions when $U=9 \mathrm{~m} / \mathrm{s}$.

3.2. Influence of the Fluid Density on the Galloping Amplitude. Although the air density has not an obvious change under 


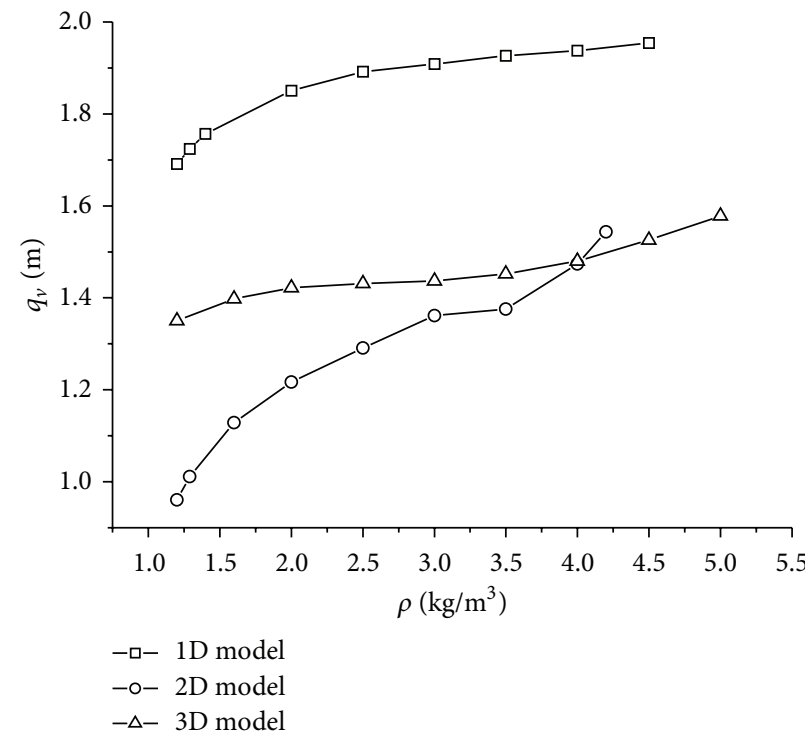

(a)

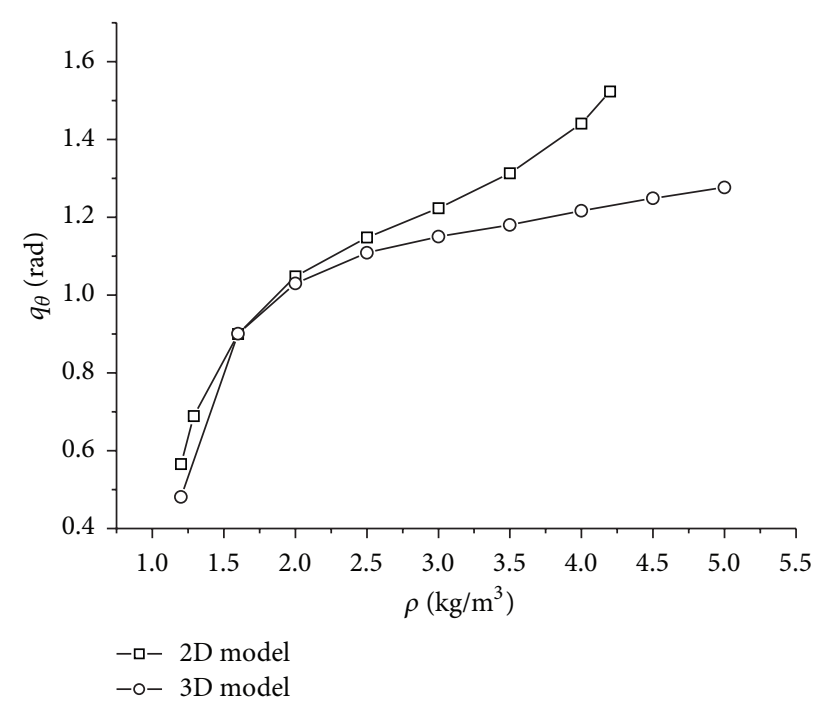

(b)

FIGURE 6: The influence of the flow density on the galloping amplitude: (a) vertical amplitude and (b) torsional amplitude.

the actual conditions, the influence of the flow density on the galloping amplitude is also studied in this paper. And the results are shown in Figure 6. Figure 6(a) presents the vertical amplitude versus the flow density.

In the given flow density interval $[1,5] \mathrm{kg} / \mathrm{m}^{3}$, the galloping amplitudes have all increased with the increasing of the flow density based on the three models. In the same flow density, the amplitude calculated by the one-DOF model is the largest. And the amplitudes obtained by two-DOF model and three-DOF model have a little gap. So the torsional motion has an obvious influence to the galloping amplitude and the horizontal motion has not an obvious influence on the change of the flow density.

Figure 6(b) gives the torsional angle versus the flow density. From the results, we can see that the torsional angles decrease with the increasing of the flow density. And, at the same flow density, the torsional angle calculated by the twoDOF model is all but equal to the three-DOF model though the angle from the two-DOF model is slightly larger than the three-DOF model.

It must be clarified that the actual air density is approximately $1.29 \mathrm{~kg} / \mathrm{m}^{3}$. In this study, in order to analyze the effect of fluid density on the galloping amplitude in theory, the value of parameter $\rho$ is given in an interval $[1,5] \mathrm{kg} / \mathrm{m}^{3}$ as the other parameters. So the other values of the parameter $\rho$ used in this analysis have no practical significance.

\subsection{Influence of the Span Length on the Galloping Amplitude.} Figure 7 shows the galloping vertical amplitude $q_{v}$ and $q_{\theta}$ versus the span length $L$ based on three different models. In this study, the span length interval is selected as $[50,260] \mathrm{m}$, in which it is familiar that the galloping modal is presented with one loop. From Figure 7(a), we can see that the galloping amplitudes have all increased with the increasing of the span length based on the three models. At the same span length, the galloping amplitude calculated by the one-DOF model is much larger than the other models, and the amplitude calculated by the two-DOF model is the smallest. Figure 7(b) presents the torsional angle versus the span length. From the results, we can see that the torsional angles obtained by the $2 \mathrm{D}$ model and the 3D model have the same results by and large.

3.4. Influence of the Damping Ratio on the Galloping Amplitude. Figure 8 shows the galloping amplitudes $q_{v}$ and $q_{\theta}$ versus the damping ratios $\zeta_{y}$ and $\zeta_{\theta}$ based on three different models. Figure 8(a) gives the vertical amplitude versus the vertical damping ratio. In the given damping ration interval $[0,0.08]$, the galloping amplitudes have all decreased rapidly with the increasing of the damping ratio based on the three models. So it has obvious influence of the damping to the galloping amplitude. And the amplitude obtained by the three-DOF model has the most gradual decrease with the increasing of the damping ratio. Figure $8(\mathrm{~b})$ presents the torsional angle versus the torsional damping ratio. From the results, we can see that the torsional angles have an obvious decrease with the increasing of the damping ratio. And at the same damping ratio, the torsional angle calculated by the twoDOF model is all but equal to the three-DOF model though the angle obtained by the three-DOF model is slightly larger than the two-DOF model.

3.5. Influence of the Initial Tension on the Galloping Amplitude. Figure 9 shows the galloping amplitudes $q_{v}$ and $q_{\theta}$ versus the initial tension $T$ based on three different models. Figure 9(a) presents the vertical amplitude versus the initial tension. In the given tension interval $[30,90] \mathrm{kN}$, the galloping amplitude will decrease slightly with the increasing of the initial tension. And at the same tension, the galloping amplitude 


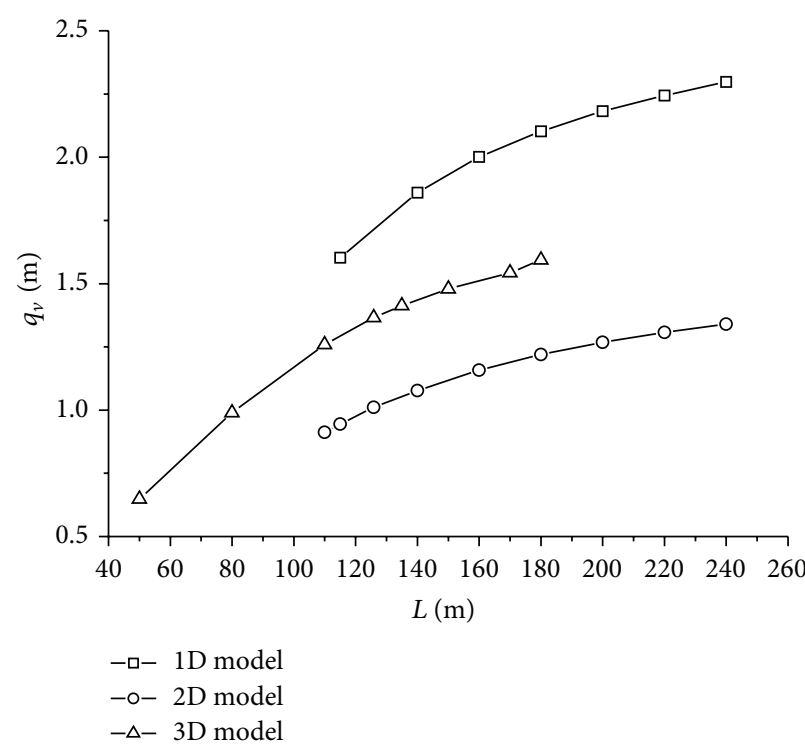

(a)

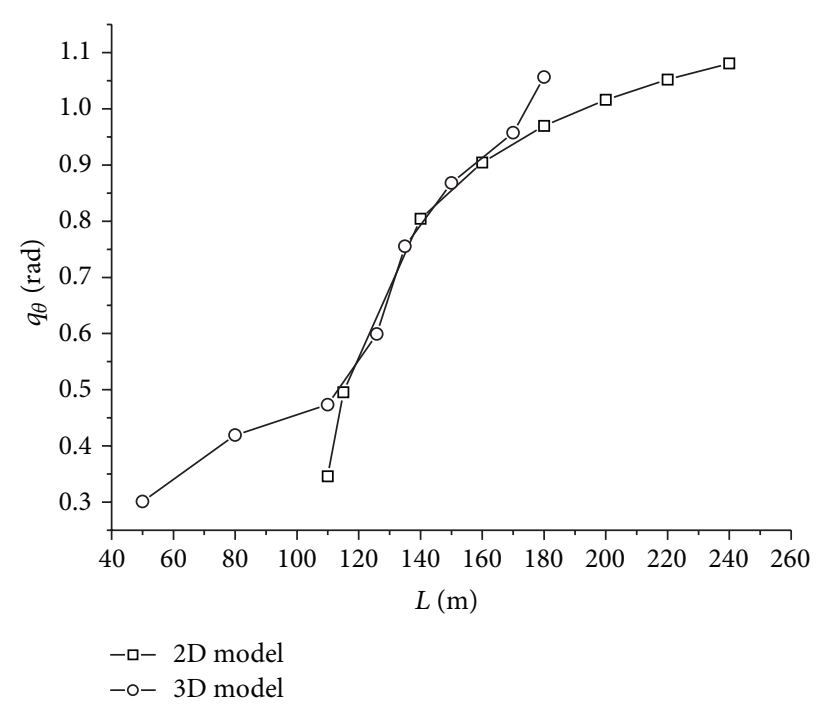

(b)

FIgURE 7: The influence of the span length on the galloping amplitude: (a) vertical amplitude and (b) torsional amplitude.

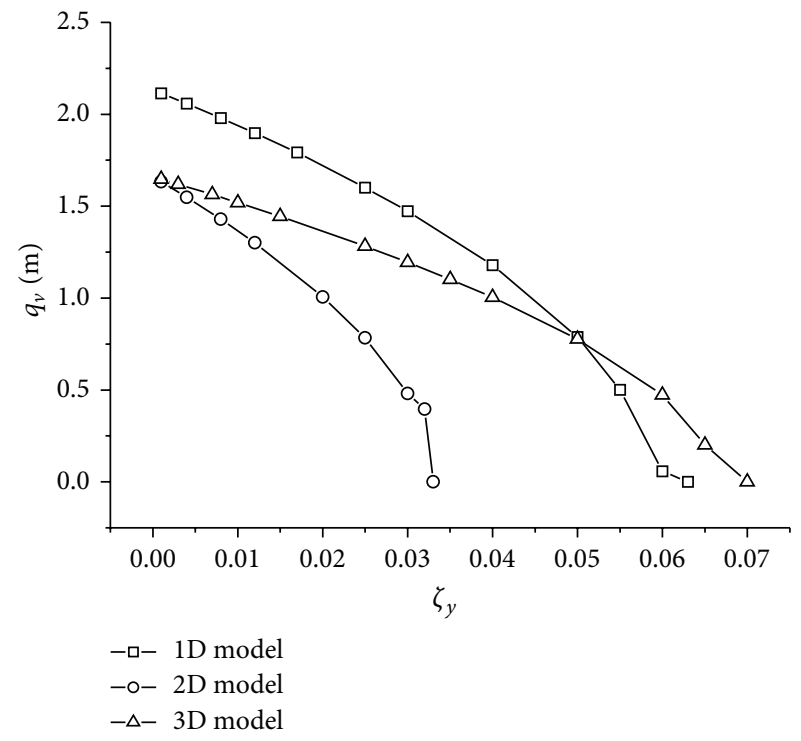

(a)

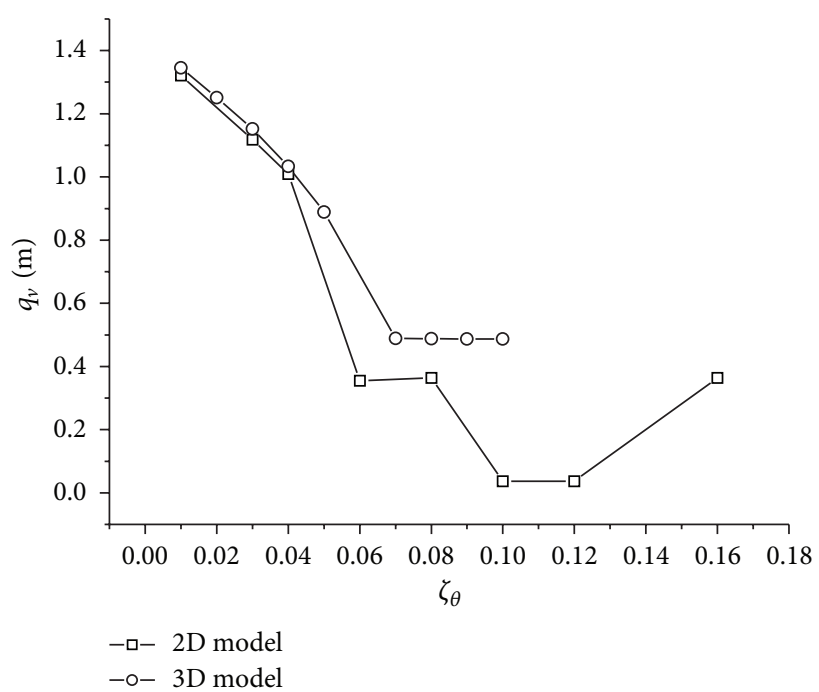

(b)

FIGURE 8: The influence of the damping ratio on the galloping amplitude: (a) vertical amplitude and (b) torsional amplitude.

calculated by the one-DOF model is much larger than the other models, and the amplitude calculated by the two-DOF model is the smallest.

Figure 9(b) gives the torsional angle versus the initial tension. From the results, we can see that it has an obvious difference between the two-DOF model and the three-DOF model. In the given tension interval, it has no obvious change of the initial tension to the torsional angle calculated by two-DOF model. But the torsional angle obtained by three-DOF model has a remarkable decrease as the tension increases. So the horizontal motion has an obvious influence on the torsional motion on the conductor galloping under the condition of different tensions.

\section{Conclusions}

In this paper, one-DOF, two-DOF, and three-DOF models on the iced conductor galloping were proposed based on the continuum cable model. The contrast with the three mathematical models of different DOFs was also studied by 


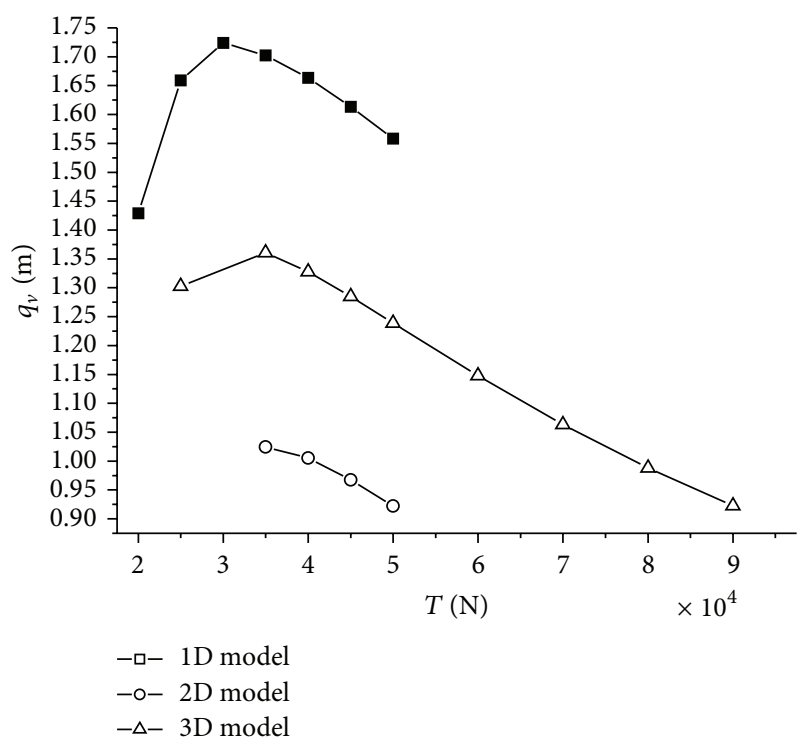

(a)

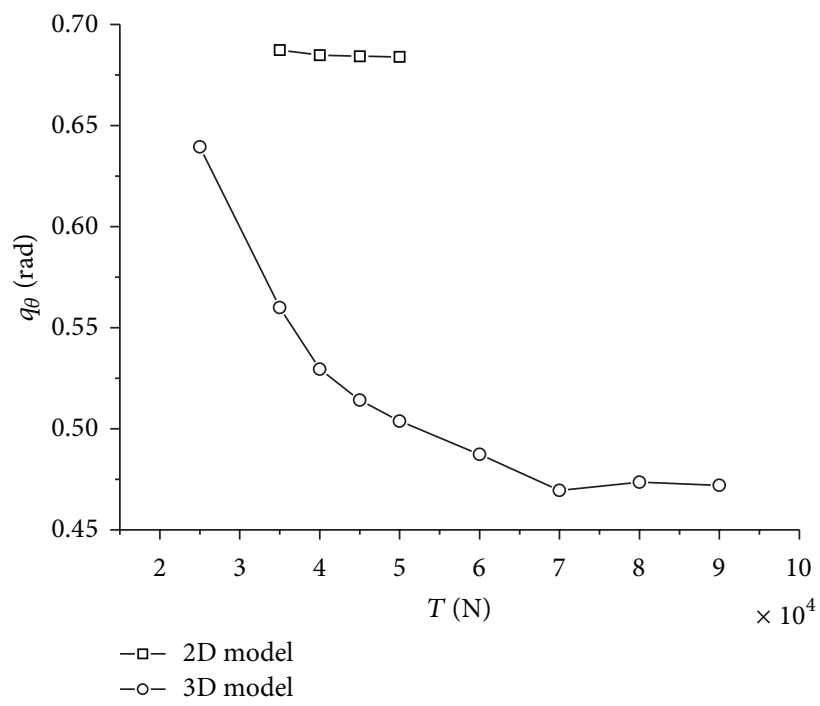

(b)

FIgURE 9: The influence of the initial tension on the galloping amplitude: (a) vertical amplitude and (b) torsional amplitude.

analyzing the galloping amplitude obtained under different factors. The obtained results could be summarized as follows.

(1) These three models can all present the trend of galloping amplitude change relative to the factors from the point view of qualitative analysis.

(2) At the same value of the influence factor, the galloping vertical amplitude calculated by the one-DOF model is much larger than the other models. So the accessorial coupling of the horizontal and torsional motions with the vertical motion will reduce the galloping vertical amplitude. And the vertical amplitude obtained by the three-DOF model is larger than the two-DOF model due to the effect of the horizontal motion.

(3) The torsional motion amplitude on the conductor galloping has an obvious influence on the change of the given factors. It is more sensitive for the torsional angle obtained by the three-DOF model than the twoDOF model with the change of the initial tension. And there is no obvious difference between the two models for other influence factors.

(4) It is more conspicuous for the wind velocity and the damping ratio to influence the galloping vertical amplitude. Actually, the vertical amplitude is the most important characteristic on the conductor galloping to cause the damages of transmission lines. So it will be an effective method to control the galloping phenomenon by modifying the aerodynamic property and the damping ratio of the conductors in the antigalloping design.

\section{Conflict of Interests}

The authors declare that there is no conflict of interests regarding the publication of this paper.

\section{Acknowledgment}

The authors are grateful for the financial support from the National Natural Science Foundation of China (51008288 and 51305411).

\section{References}

[1] Cigre SCB2 WG11 and Task Force 02.11.06, "State of the art of conductor galloping," Electra No 322, 2007, Convenor: J. L. Lilien.

[2] R. D. Blevins, Flow-Induced Vibration, Van Nostrand Reinhold, New York, NY, USA, 1990.

[3] Y. L. Guo, G. X. Li, and C. Y. You, Galloping of the Transmission Line, China Electronic Power Press, Beijing, China, 2003, (Chinese).

[4] C. B. Rawlins, "Transmission line reference book-wind-induced conductor motion," in Galloping Conductors, vol. 792, chapter 4, EPRI Research Project, 1979.

[5] J. P. den Hartog, "Transmission line vibration due to sleet," Transactions of AIEE, vol. 51, pp. 1074-1086, 1932.

[6] O. Nigol and P. G. Buchan, "Conductor galloping part I: den hartog mechanism," IEEE transactions on power apparatus and systems, vol. 100, no. 2, pp. 699-707, 1981.

[7] O. Nigol and P. G. Buchan, "Conductor galloping part II: torsional mechanism," IEEE Transactions on Power Apparatus and Systems, vol. 100, no. 2, pp. 708-720, 1981. 
[8] P. Yu, A. H. Shah, and N. Popplewell, "Inertially coupled galloping of iced conductors," Journal of Applied Mechanics, Transactions ASME, vol. 59, no. 1, pp. 140-145, 1992.

[9] P. Yu, M. Desai, A. H. Shah, and N. Popplewell, "Three-degreeof-freedom model for galloping. Part I: formulation," Journal of Engineering Mechanics, vol. 119, no. 12, pp. 2404-2425, 1993.

[10] A. Luongo, G. Rega, and F. Vestroni, "Planar non-linear free vibrations of an elastic cable," International Journal of NonLinear Mechanics, vol. 19, no. 1, pp. 39-52, 1984.

[11] A. Luongo and G. Piccardo, "Non-linear galloping of sagged cables in 1:2 internal resonance," Journal of Sound and Vibration, vol. 214, no. 5, pp. 915-936, 1998.

[12] C. L. Lee and N. C. Perkins, "Nonlinear oscillations of suspended cables containing a two-to-one internal resonance," Nonlinear Dynamics, vol. 3, no. 6, pp. 465-490, 1992.

[13] K. G. McConnell and C.-N. Chang, "A study of the axialtorsional coupling effect on a sagged transmission line," Experimental Mechanics, vol. 26, no. 4, pp. 324-329, 1986.

[14] W. N. White, P. M. Lynch, C. D. Huang, and S. Venkatasubramanian, "The equations of motion for the torsional and bending vibrations of a stranded cable," Journal of Applied Mechanics, vol. 59, no. 2, pp. S224-S229, 1992.

[15] A. Luongo, D. Zulli, and G. Piccardo, "A linear curved-beam model for the analysis of galloping in suspended cables," Journal of Mechanics of Materials and Structures, vol. 2, no. 4, pp. 675694, 2007.

[16] G. Diana, S. Bruni, F. Cheli, F. Fossati, and A. Manenti, "Dynamic analysis of the transmission line crossing 'Lago de Maracaibo," Journal of Wind Engineering and Industrial Aerodynamics, vol. 74-76, pp. 977-986, 1998.

[17] C.-L. Lu and N. C. Perkins, "Nonlinear spatial equilibria and stability of cables under uni-axial torque and thrust," Journal of Applied Mechanics, Transactions ASME, vol. 61, no. 4, pp. 879886, 1994.

[18] A. Luongo, D. Zulli, and G. Piccardo, "Analytical and numerical approaches to nonlinear galloping of internally resonant suspended cables," Journal of Sound and Vibration, vol. 315, no. 3, pp. 375-393, 2008.

[19] A. Luongo, D. Zulli, and G. Piccardo, "On the effect of twist angle on nonlinear galloping of suspended cables," Computers and Structures, vol. 87, no. 15-16, pp. 1003-1014, 2009.

[20] A. Luongo and D. Zulli, "Dynamic instability of inclined cables under combined wind flow and support motion," Nonlinear Dynamics, vol. 67, no. 1, pp. 71-87, 2012.

[21] Z. H. Qin, Y. S. Chen, X. P. Zhan, B. Liu, and K. J. Zhu, "Research on the galloping and anti-galloping of the transmission line," International Journal of Bifurcation and Chaos, vol. 22, no. 2, Article ID 1250038, 34 pages, 2012.

[22] S. M. Meng, W. Kong, and G. Q. Liu, Design on Overhead Transmission Lines, China Electric Power Press, Beijing, China, 2007.

[23] P. McComber and A. Paradis, "A cable galloping model for thin ice accretions," Atmospheric Research, vol. 46, no. 1-2, pp. 13-25, 1998.

[24] R. W. Clough and J. Penzien, Dynamics of Structures, McGrawHill, New York, NY, USA, 1975.

[25] H. M. Irvine and T. K. Caughey, "The linear theory of free vibrations of a suspended cables," Proceeding of the Royal Society of London A, vol. 341, pp. 299-315, 1974.

[26] L. Wang and G. Rega, "Modelling and transient planar dynamics of suspended cables with moving mass," International Journal of Solids and Structures, vol. 47, no. 20, pp. 2733-2744, 2010.
[27] G. A. Vio, G. Dimitriadis, and J. E. Cooper, "Bifurcation analysis and limit cycle oscillation amplitude prediction methods applied to the aeroelastic galloping problem," Journal of Fluids and Structures, vol. 23, no. 7, pp. 983-1011, 2007. 

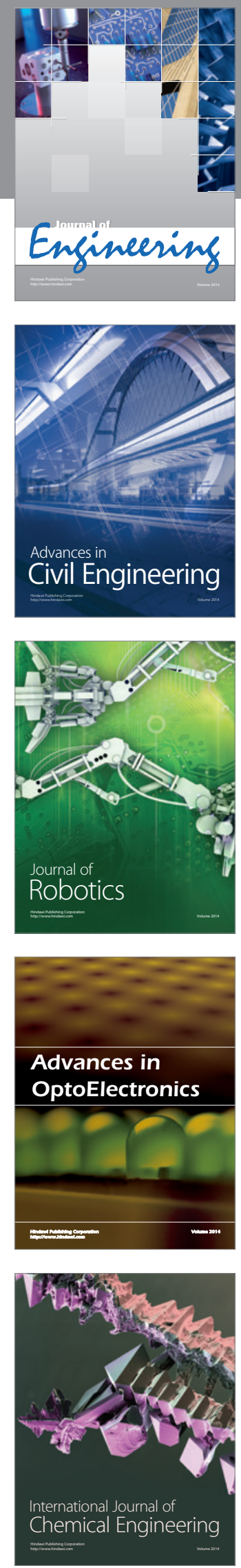

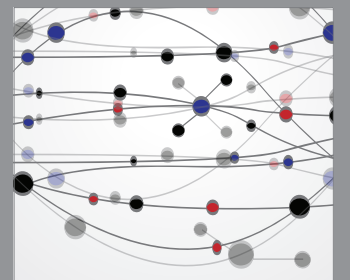

The Scientific World Journal
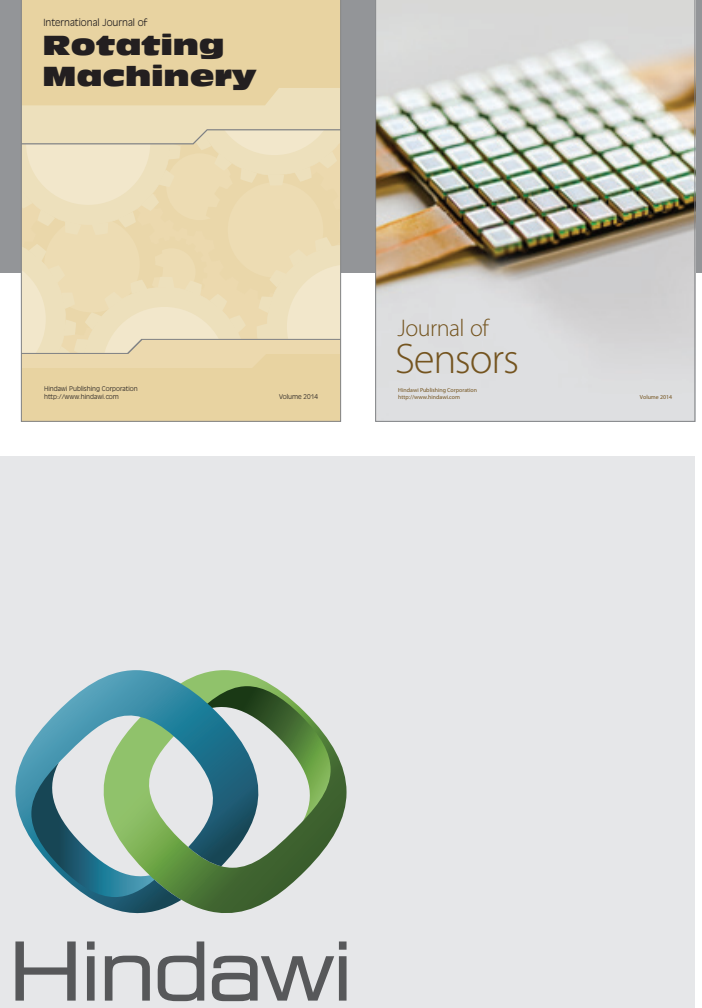

Submit your manuscripts at http://www.hindawi.com
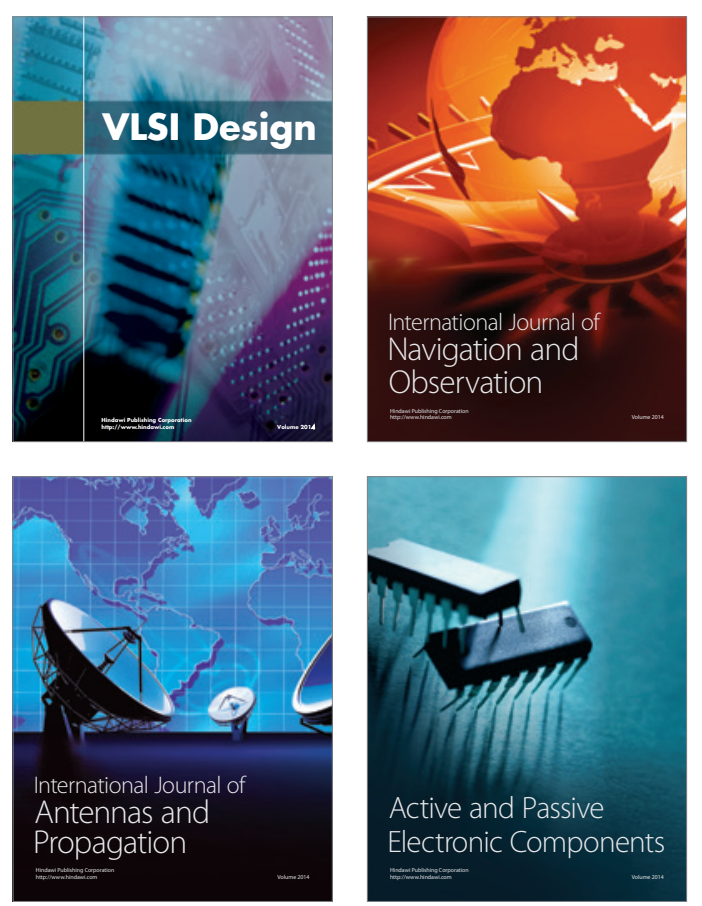
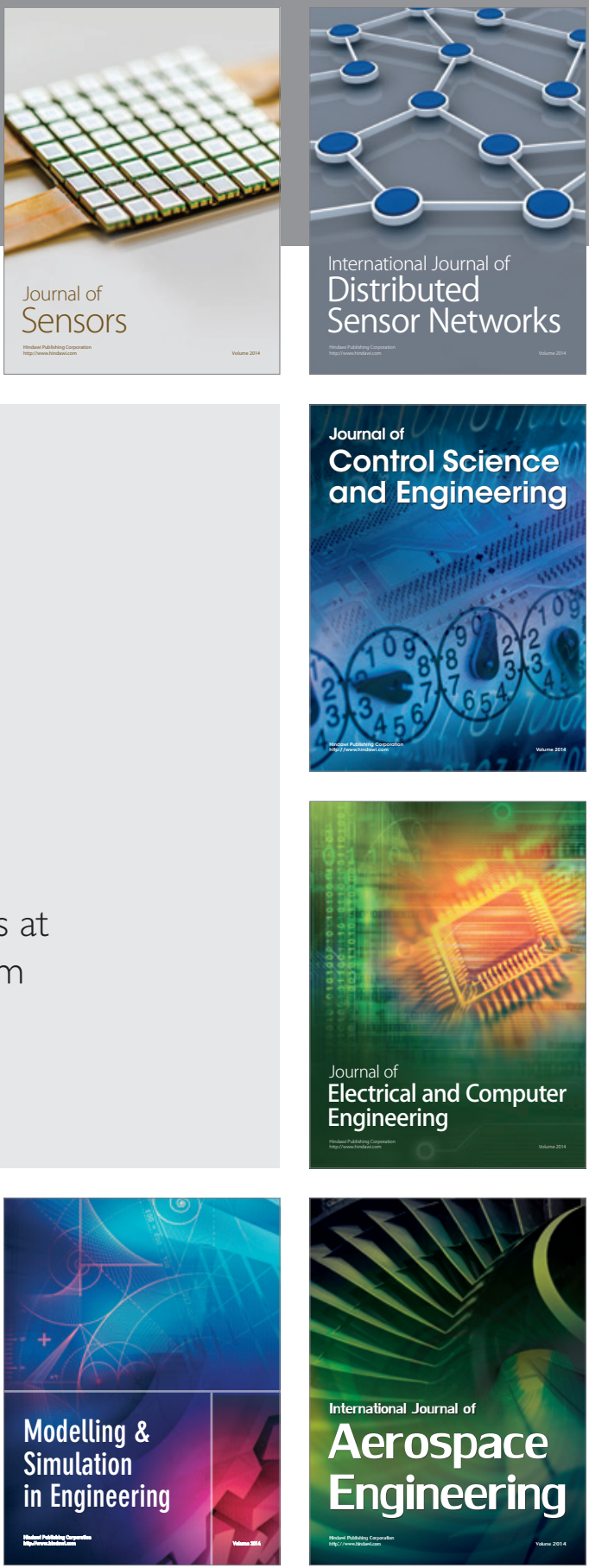

Journal of

Control Science

and Engineering
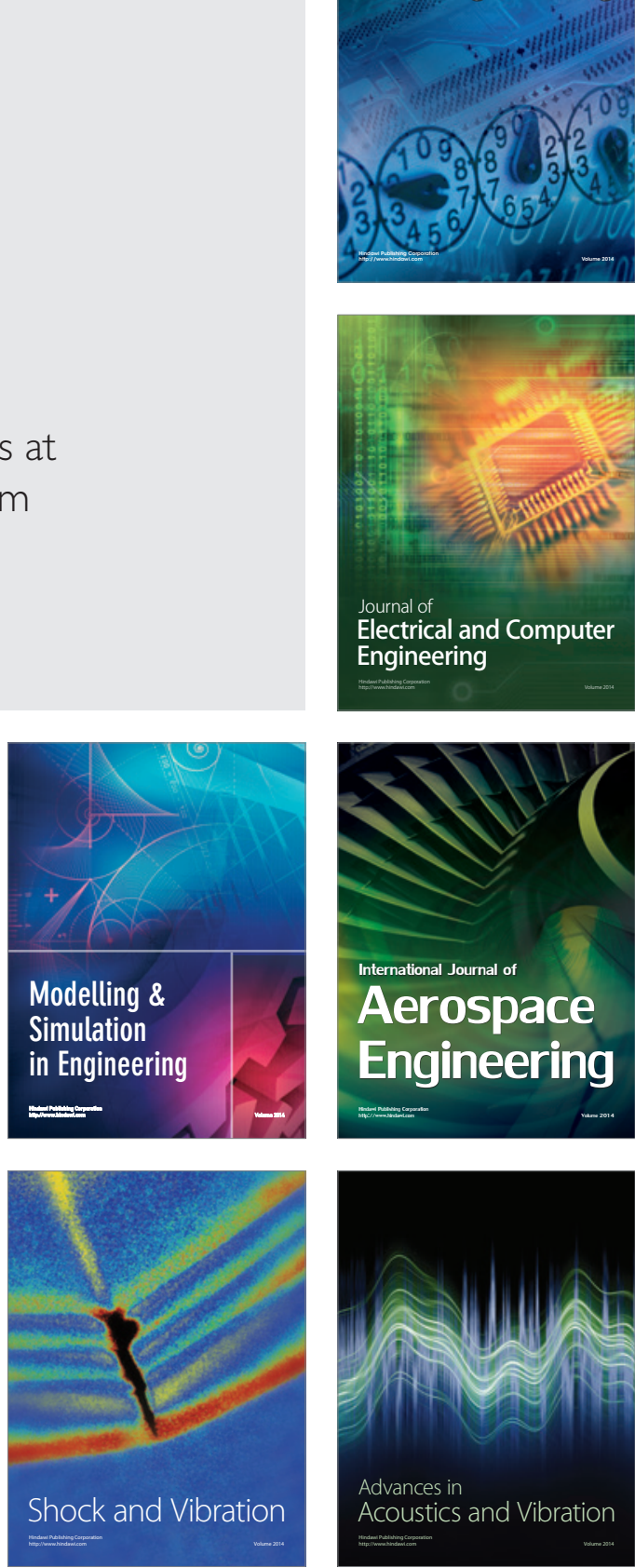